\title{
The nucleon microscopic optical potential based on the Skyrme interaction
}

\author{
Yongli Xu ${ }^{1, a}$, Hairui Guo ${ }^{2}$, Yinlu Han ${ }^{3, b}$, and Qingbiao Shen $^{3}$ \\ ${ }^{1}$ College of Physics and Electronic Science, Shanxi Datong University, Datong 037009, P.R. China \\ 2 Institute of Applied Physics and Computational Mathematics, Beijing 100094, P.R. China \\ 3 Key Laboratory of Nuclear Data, China Institute of Atomic Energy, PO Box (275-41), Beijing 102413, P.R. China
}

\begin{abstract}
The nucleon microscopic optical potential based on the conventional and extended Skyrme interactions are achieved by the single-particle Green function method through nuclear matter approximation and local density approximation. The nucleon-nucleon scattering observables are calculated by the obtained microscopic optical potential and the results are compared with the corresponding experimental data. Good agreement is generally obtained between them.
\end{abstract}

\section{Introduction}

The microscopic optical potential (MOP) is of the fundamental importance in the field of the nuclear physics and astrophysics. It can not only provide us some information on the nuclear structure, but also give some theoretical and experimental guidance for nuclear reaction. Moreover, an important feature of a good MOP is that it can be used to reliably predict these observables in the energy and mass number range where no experimental measurement data exist.

According to the many-body theory, the nucleon MOP can be equivalent to the mass operator of the singleparticle Green function [1,2]. Furthermore, the first-order mass operator and the imaginary part of the second-order mass operator are respectively taken as the real part and imaginary part of the nucleon MOP in the nuclear matter. The real and imaginary parts of the MOP for finite nuclei are obtained by applying a local density approximation [3]. Using certain versions of the Skyrme interactions we have obtained the microscopic optical potential [4] for finite nuclei which showed that the potential depth, shape, relative contributions of the surface and volume parts, as well as the energy dependence are in reasonable agreement with the phenomenological optical potentials and those based on realistic nuclear force for certain energy regions.

In this present work, the nucleon MOP based on the conventional and extended Skyrme interactions is obtained. The total cross sections, nonelastic cross sections, elastic scattering angular distributions, and analyzing powers are calculated by the obtained nucleon MOP for neutron induced reactions below $100 \mathrm{MeV}$. The calculated results are further compared with the available experimental data.

Section 2 provides a description of the theoretical models and methods. Section 3 gives analysis and

\footnotetext{
a e-mail: xuyongli776@126.com

b e-mail: hanyl@ciae.ac.cn
}

comparisons of calculated results with experimental data. Finally, Sect. 4 gives our conclusions.

\section{The theoretical models and methods}

The Hamiltonian of the system composed of an incident particle and a target nucleus, which refers only to the twobody interaction, can be expressed as

$$
H=H_{0}+H_{1},
$$

where

$$
\begin{gathered}
H_{0}=\sum_{i}\left(t_{i}+U_{i}\right), \\
H_{1}=\frac{1}{2 !} \sum_{i \neq j} V_{i j}-\sum_{i} U_{i},
\end{gathered}
$$

$H_{0}$ is the single-particle Hamiltonian, $H_{1}$ is the residual interaction, and $U_{i}$ is the mean field of the single particle.

By the Green function method, the nucleon MOP is equivalent to the mass operator $M_{\alpha, \alpha}$ of the single-particle Green function up to the second order Goldstone diagram. Figure 1 depicts the first and second-order Feynman diagrams of the single-particle Green function.

Moreover, the first-order mass operator has the following form

$$
M_{\alpha, \alpha}^{(1)}=\sum_{\rho} V_{\alpha \rho, \alpha \rho} n_{\rho},
$$

where $\rho=1$ and 0 denote below and above the Fermi surface, respectively. The matrix elements $V_{\alpha \rho, \alpha \rho}$ is

$$
V_{\alpha \rho, \alpha \rho}=\langle\alpha \rho|V| \alpha \rho\rangle_{A},
$$

where $A$ denotes the anti-symmetrization.

The second-order mass operator is

$$
M_{\alpha, \alpha}^{(2)}(\omega)=\frac{1}{2} \sum_{\lambda \mu \nu} \frac{\left(1-n_{\lambda}\right)\left(1-n_{\mu}\right) n_{v}}{\varepsilon_{\alpha}+\varepsilon_{v}-\varepsilon_{\lambda}-\varepsilon_{\mu}+i \eta} V_{\alpha \nu, \lambda \mu} V_{\lambda \mu, \alpha \nu}
$$

(C) The Authors, published by EDP Sciences. This is an Open Access article distributed under the terms of the Creative Commons Attribution License 4.0 (http://creativecommons.org/licenses/by/4.0/). 


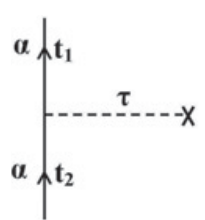

(a)

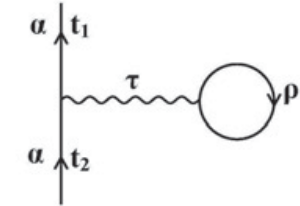

(b)

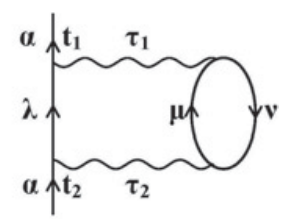

(c)
Figure 1. The first and second-order Feynman diagrams of the one-particle Green function. The dashed line represents the mean field.

where $\varepsilon$ is the energies corresponding to different particles, and $\eta$ is an infinitesimal.

Under the mean-field approximation, the first-order mass operator $M_{\alpha, \alpha}^{(1)}$ represents the real part of the MOP. The imaginary part of the second-order mass operator $M_{\alpha, \alpha}^{(2)}$ is taken as the imaginary part of the MOP because the second order diagrams are the lowest order diagrams to contribute to the imaginary part of the MOP.

The two-body effective interaction $V$ in Eq. (3) is given by the extended Skyrme interaction [4], which is expressed as

$$
\begin{aligned}
V_{1,2}(\vec{R}, \vec{r})= & t_{0}\left(1+x_{0} P^{\sigma}\right) \delta(\vec{r})+t_{2}\left(1+x_{2} P^{\sigma}\right) \vec{k}^{\prime} \cdot \delta(\vec{r}) \vec{k} \\
& +\frac{1}{2} t_{1}\left(1+x_{1} P^{\sigma}\right)\left(\vec{k}^{\prime 2} \delta(\vec{r})+\delta(\vec{r}) \vec{k}^{2}\right) \\
& +\frac{1}{6} t_{3}\left(1+x_{3} P^{\sigma}\right) \rho^{\alpha}(\vec{R}) \delta(\vec{r})+\frac{1}{2} t_{4}\left(1+x_{4} P^{\sigma}\right) \\
& \times\left(\vec{k}^{\prime 2} \rho^{\alpha_{4}} \delta(\vec{r})+\delta(\vec{r}) \rho^{\alpha_{4}} \vec{k}^{2}\right)+t_{5}\left(1+x_{5} P^{\sigma}\right) \vec{k}^{\prime} \\
& \cdot \rho^{\alpha_{5}} \delta(\vec{r}) \vec{k}+i W_{0}\left(\vec{\sigma}_{1}+\vec{\sigma}_{2}\right) \vec{k}^{\prime} \times \delta(\vec{r}) \vec{k},
\end{aligned}
$$

where $\vec{r}=\vec{r}_{1}-\vec{r}_{2}$ and $\vec{R}=\frac{1}{2}\left(\vec{r}_{1}+\vec{r}_{2}\right)$ are the relative and center-of-mass radius vectors of two nucleons, $\vec{k}=\frac{1}{2 i}\left(\vec{\nabla}_{1}-\vec{\nabla}_{2}\right)$ acting on the right and its Hermitian conjugate operator $\vec{k}^{\prime}=-\frac{1}{2 i}\left(\vec{\nabla}_{1}-\vec{\nabla}_{2}\right)$ acting on the left are respectively the operators of momentum of the nucleon relative motion in the initial and final states, $P^{\sigma}$ is the spin exchange operator and $\vec{\sigma}$ is the Pauli spin matrix. If the $t_{4}$ and $t_{5}$ are zero, the expression of Eq. (7) is the so-called conventional Skyrme interaction $[4,5]$.

We use the nuclear matter approach. In the nuclear matter, the wave function of nucleon $\alpha$ is the plane wave. Based on the conventional and extended Skyrme interaction parameters, the real and imaginary parts of nucleon MOP can be achieved and the detailed description can be found in Refs. [4,5].

For a finite nucleus MOP, the simplest way is to use the local density approximation(LDA) [3]. As in the approach used in Refs. $[4,6,7]$, the nuclear densities are similarly expressed by the Negele's empirical formula [8].

The real part of spin-orbit potential obtained by the Skyrme-Hartree-Fock approach is amended by the results of relativistic microscopic optical potential, which is considered the correlation with incident energy [5]. The imaginary part of spin-orbit potential below $100 \mathrm{MeV}$ is usually very small and ignored here.

\section{Results and discussions}

Based on the nucleon MOP obtained with the existing Skyrme interaction parameters, the total cross sections, nonelastic cross sections, elastic scattering angular
Table 1. The obtained extended Skyrme interaction SkC16.

\begin{tabular}{lc}
\hline \hline$t_{0}\left(\mathrm{MeV} \cdot \mathrm{fm}^{3}\right)$ & -1474.496 \\
$t_{1}\left(\mathrm{MeV} \cdot \mathrm{fm}^{5}\right)$ & 1845.451 \\
$t_{2}\left(\mathrm{MeV} \cdot \mathrm{fm}^{5}\right)$ & -553.180 \\
$t_{3}\left(\mathrm{MeV} \cdot \mathrm{fm}^{3+3 \alpha}\right)$ & 10275.362 \\
$t_{4}(\mathrm{MeV} \cdot \mathrm{fm})$ & -2040.471 \\
$t_{5}(\mathrm{MeV} \cdot \mathrm{fm})$ & 575.278 \\
$x_{0}$ & 0.475 \\
$x_{1}$ & 0.588 \\
$x_{2}$ & 0.277 \\
$x_{3}$ & 1.009 \\
$x_{4}$ & 0.764 \\
$x_{5}$ & 0.623 \\
$\alpha$ & 0.516 \\
$\alpha_{4}$ & 0.197 \\
$\alpha_{5}$ & 0.191 \\
$\mathrm{~W}_{0}\left(\mathrm{MeV} \cdot \mathrm{fm}^{5}\right)$ & 100.000 \\
\hline \hline
\end{tabular}

distributions, and analyzing powers are calculated below $100 \mathrm{MeV}$. The results calculated using the obtained nucleon MOP based on the extended Skyrme interaction GS2 [9] are closest to the corresponding experimental data. But the GS2 can not well describe the properties of nuclear matter and ground-state of finite nuclei.

For a unified description of the nuclear properties, We thus constructed the new conventional Skyrme interaction SkC [5] and extended Skyrme interaction SkC16 which listed in Table 1. They are achieved by simultaneously fitting the nuclear matter properties and ground-state properties of finite nuclei, as well as the neutron-nucleus scattering observables including the total cross sections, nonelastic cross sections, elastic scattering angular distributions and analyzing powers in the mass number range $24 \leqslant \mathrm{~A} \leqslant 209$ below $100 \mathrm{MeV}$. Moreover, the neutron induced reactions for light and actinide nuclei are predicted by the obtained Skyrme interaction SkC below $100 \mathrm{MeV}$, as well as for too deformed targets in the mass number range $148 \leqslant \mathrm{~A} \leqslant 194$ [10]. All of the theoretical results give reasonable agreements with the corresponding experimental data.

At the present time, we obtain the nucleon MOP using the Skyrme interactions $\mathrm{SkC}$ and $\mathrm{SkC16}$. The total cross sections, nonelastic cross sections, elastic scattering angular distributions, and analyzing powers are calculated by the obtained nucleon MOP in the mass number range $24 \leqslant A \leqslant 209$ at incident neutron energies below $100 \mathrm{MeV}$. The calculated results are compared with the available experimental data which are from EXFOR at http: //www-nds.iaea.org/exfor/exfor.htm.

Figure 2 presents the total cross sections and nonelastic cross sections calculated using the new extended Skyrme interaction $\mathrm{SkC} 16$ for ${ }^{27} \mathrm{Al}$, which are compared with those of calculations based on the conventional Skyrme interaction $\mathrm{SkC}$ and extended Skyrme interaction GS2. For the total cross sections of light targets, the narrow and broad resonances with large amplitudes appear in the experiment data for the incident energy range from hundreds of $\mathrm{keV}$ to few $\mathrm{MeV}$. It is just the shaded area in Fig. 2(a), where the calculations can only provide smooth average results in this region. Above $3 \mathrm{MeV}$, we get the usual agreement with data, and the deviation of our calculations from the experiment are on average within $5 \%$ for ${ }^{27} \mathrm{Al}$. For the nonelastic cross sections, most of the 

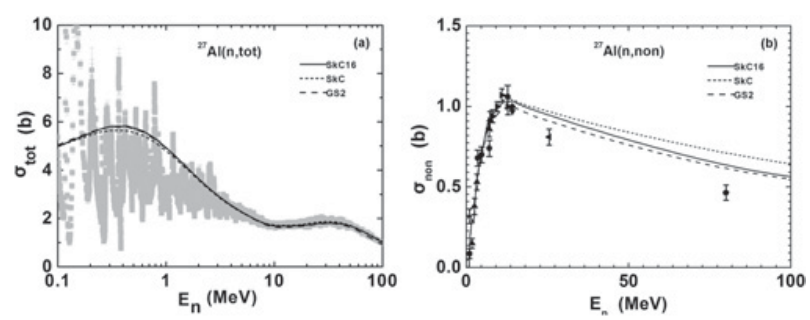

Figure 2. Calculated total and nonelastic cross sections compared with the experimental data for $n+{ }^{27} \mathrm{Al}$ reaction.

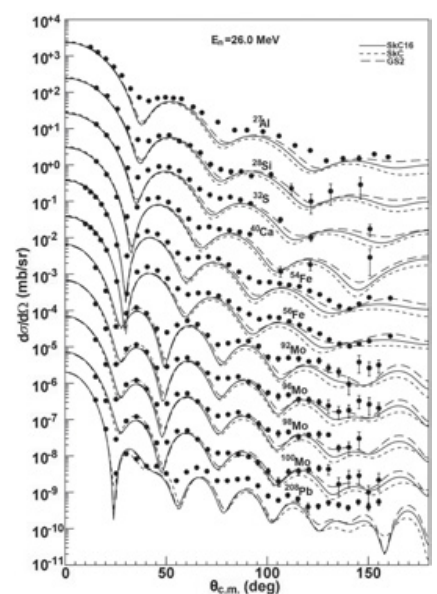

Figure 3. Calculated elastic scattering angular distributions compared with the experimental data at incident neutron energies 26.0 MeV.

experiment of nonelastic cross sections are made below $40 \mathrm{MeV}$. As is shown in Fig. 2(b), the shapes of nonelastic cross sections calculated with these Skyrme interactions are similar with the experimental data for ${ }^{27} \mathrm{Al}$, while the calculations with SkC16 and GS2 are much closer to the experimental data.

The elastic scattering angular distributions are calculated for different targets (from ${ }^{24} \mathrm{Mg}$ to ${ }^{208} \mathrm{~Pb}$ ) below $100 \mathrm{MeV}$. These results are compared with those of calculations based on the SkC and GS2. Figure 3 presents these calculated results for different targets at incident neutron energy $26.0 \mathrm{MeV}$ together with the experimental data. One can see that the new extended Skyrme interaction SkC16 improves the calculations compared with the conventional Skyrme interaction $\mathrm{SkC}$ in the region of large scattering angles, namely above 80 degree. The results calculated using the obtained MOP based on the SkC16 and GS2 are fairly close to each other. For the reason, below we shall restrict ourselves to the consideration of calculations with only the new extended Skyrme interaction $\mathrm{SkC16.}$

The elastic scattering angular distributions are calculated using the obtained MOP based on the new extended Skyrme interaction $\mathrm{SkC} 16$ for ${ }^{27} \mathrm{Al}$ below $100 \mathrm{MeV}$. As shown in Fig. 4, the good agreements are obtained except for $15.43,16.88$, and $18.0 \mathrm{MeV}$, where there is a slight underestimation for the extreme value and the curve shapes of our results is similar with experimental data.

In Figs. 5 and 6, we present the elastic scattering angular distributions calculated for ${ }^{98} \mathrm{Mo}$ and ${ }^{120} \mathrm{Sn}$ below $100 \mathrm{MeV}$. It can be clearly seen that the calculated results give a satisfactory agreement with experimental data.
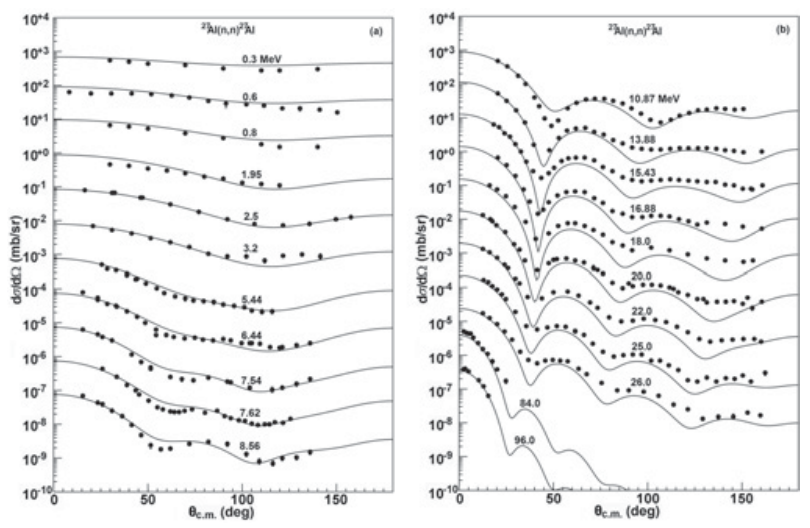

Figure 4. Calculated elastic scattering angular distributions compared with the experimental data for $n+{ }^{27} \mathrm{Al}$ reaction.
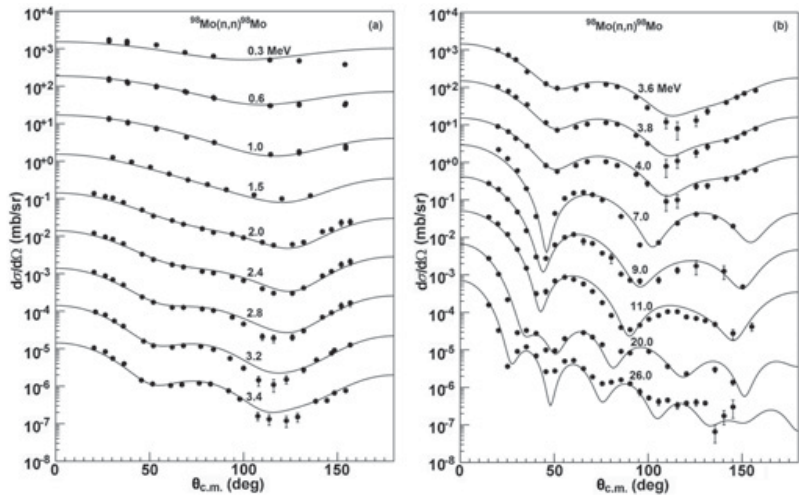

Figure 5. Same as Fig. 4 but for $n+{ }^{98}$ Mo reaction.
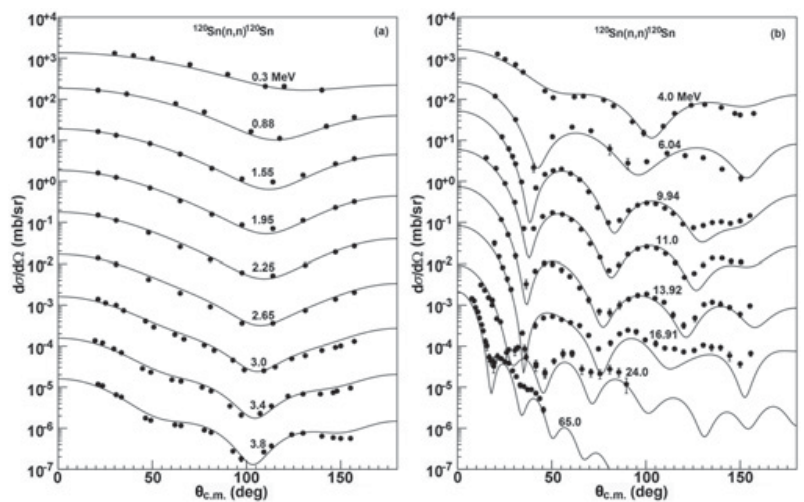

Figure 6. Same as Fig. 4 but for $n+{ }^{120} \mathrm{Sn}$ reaction.

As the calculations of the elastic scattering differential cross sections, we present the example of the analyzing powers $A_{y}(\theta)$ for ${ }^{58} \mathrm{Ni}$ and ${ }^{120} \mathrm{Sn}$ below $100 \mathrm{MeV}$, as is shown in Fig. 7. Obviously, the obtained MOP provide a satisfactory description of the experimental analyzing powers.

We also make a prediction with the obtained MOP based on the SkC16 for the neutron induced actinide nuclei reactions below $100 \mathrm{MeV}$. In Fig. 8, we present the comparisons between the elastic scattering angular distributions and the corresponding experimental data for ${ }^{232} \mathrm{Th}$. It can be seen from the figure that the largest deviations occur in the first minimum for ${ }^{232} \mathrm{Th}$, which is the reason that the experimental data include the elastic and inelastic scattering. 


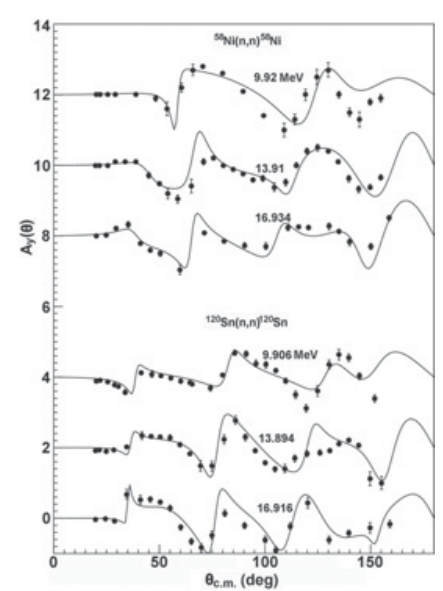

Figure 7. Calculated analyzing powers compared with the experimental data for $n+{ }^{58} \mathrm{Ni}$ and ${ }^{120} \mathrm{Sn}$ reactions.

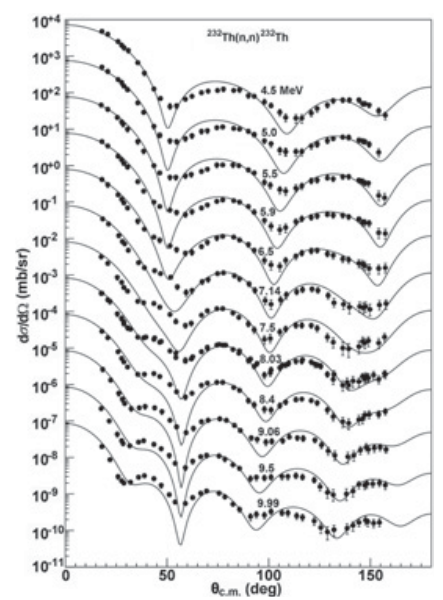

Figure 8. Same as Fig. 4 but for $n+{ }^{232}$ Th reaction.

Besides, the reaction cross sections, elastic scattering angular distributions, and analyzing powers are also predicted using the obtained MOP below $100 \mathrm{MeV}$ for proton-nucleon scattering. The calculations are make a comparison with the corresponding experimental data. The satisfactory agreement is achieved which is similar with the case of neutron-nucleon scattering because the
Coulomb potential of the nucleus are considered during the process of achieving nucleon MOP.

\section{Conclusions}

The nucleon MOP has been obtained by Green function method through nuclear matter approximation and local density approximation based on the Skyrme nucleon-nucleon effective interaction. The new extended Skyrme interaction SkC16 are adopted to achieve the nucleon MOP. Base on the obtained MOP, the total cross sections, nonelastic cross sections, elastic scattering angular distributions, and analyzing powers are calculated for different targets below $100 \mathrm{MeV}$. All of the results are satisfactory agreement with the corresponding experimental data. The performed calculations show that the obtained nucleon MOP in this paper has a good prediction power and can provide a reliable theoretical basis for the experimental research of far from $\beta$ stability nuclide.

The work was supported by the National Natural Science Foundation of China under Grants Nos. 11347175, 11405099, 11175260, 11575291.

\section{References}

[1] J.S. Bell and E.J. Squires, Phys. Rev. Lett. 3, 96 (1959)

[2] M.K. Weigel, Microscopic Optical Potentials (Springer Berlin, Heidelberg, 1979), p. 56

[3] J.W. Negele and D. Vautherin, Phys. Rev. C 5, 1472 (1972)

[4] Q.B. Shen, Y.L. Han, and H.R. Guo, Phys. Rev. C 80, 024604 (2009)

[5] Y.L. Xu, H.R. Guo, Y.L. Han and Q.B. Shen, J. Phys. G 41, 015101 (2014)

[6] J.P. Jeukenne, A. Lejeune, and C. Mahaux, Phys. Rev. C 16, 80 (1977)

[7] Q.B. Shen, J. S. Zhang, Y. Tian, et al., Z. Phys. A 303, 69 (1981)

[8] J.W. Negele, Phys. Rev. C 1, 1260 (1970)

[9] S. Krewald, V. Klemt, J. Speth, and A. Faessler, Nucl. Phys. A 281, 166 (1977)

[10] Y.L. Xu, H.R. Guo, Y.L. Han and Q.B. Shen, Int. J. Mod. Phys. E 25, 1650013 (2015) 